\title{
Association Between Thyroid Cancer and Cardiovascular Disease Risk: A Nationwide Observation Study
}

\section{Ming-Chieh Tsai}

Taipei Mackay Memorial Hospital

Cheng-Tzu Hsieh

National Taiwan University Hospital

Hsin-Yin Hsu

Taipei MacKay Memorial Hospital

\section{Tzu-Lin Yeh}

Hsinchu MacKay Memorial Hospital

\section{Wen-Chung Lee}

National Taiwan University Hospital

\section{Chun-Ju Chiang}

National Taiwan University Hospital

\section{Bo-Yu Hsiao}

National Taiwan University Hospital

Jing-Rong Jhuang

National Taiwan University Hospital

Wen-Hsuan Tsai

Taipei Mackay Memorial Hospital

\section{Shih-Ping Cheng}

Taipei Mackay Memorial Hospital

\section{Chao-Liang Chou}

Taipei Mackay Memorial Hospital

\section{Chun-Chuan Lee}

Taipei Mackay Memorial Hospital

\section{Sung-Chen Liu}

Taipei Mackay Memorial Hospital

\section{Po-Jung Tseng}

Hsin Chu Armed Force Hospital

Kuo-Liong Chien ( $\sigma$ klchien@ntu.edu.tw)

National Taiwan University Hospital 


\section{Research Article}

Keywords: thyroid cancer, coronary heart disease, ischemic stroke, atrial fibrillation

Posted Date: November 30th, 2021

DOI: https://doi.org/10.21203/rs.3.rs-1080898/v1

License: (c) (i) This work is licensed under a Creative Commons Attribution 4.0 International License. Read Full License 


\section{Abstract}

\section{Background}

Research findings on the long-term cardiovascular risk associated with thyroid cancer and treatment are unclear. This study aimed to investigate the standardized incidence rate (SIR) of coronary heart disease (CHD), ischemic stroke (IS), and atrial fibrillation (AF) among thyroid cancer patients in comparison with the general population across different age groups.

\section{Methods}

\section{Design, setting, and participants}

This observational cohort study enrolled patients between January 1, 2011 and December 31, 2016 with follow-up until December 31, 2018. This study analyzed the data of Taiwanese thyroid cancer patients registered on the National Taiwan Cancer Registry Database, with CHD and IS.

\section{Main outcomes and measures}

SIR models were used to evaluate the association between thyroid cancer and CHD, IS, AF, and cardiovascular disease outcome, stratified by age and sex. SIR analyses were also conducted for both sexes, age groups ( $<60, \geq 60$ years), and different follow-up years.

\section{Results}

After excluding 128 individuals $<20$ years or $>85$ years and with missing index data, 4274 eligible thyroid cancer patients without CHD history, 4343 patients without IS history, and 4247 patients without AF history were included for analysis. During the median follow-up of 3.5 (1.2) years, CHD incidence was 70; IS, 30; and AF, 20. Compared with the general population, thyroid cancer patients had higher CHD risk throughout the age spectrum (SIR, 1.33; 95\% confidence interval [Cl], 1.02 to 1.65). The overall CHD risk gradually declined, but it was persistent for more than 5 years after thyroid cancer diagnosis. CHD risk was observed in both males and females, especially in young thyroid cancer patients $<65$ years. However, lower SIRs for IS (SIR, $0.74 ; 95 \% \mathrm{Cl}, 0.47$ to 1 ), AF (SIR, $0.74 ; 95 \% \mathrm{Cl}, 0.42$ to 1.06 ), and cardiovascular disease (SIR, $0.88 ; 95 \% \mathrm{Cl}, 0.7$ to 1.05 ) were observed among thyroid cancer patients (not significant).

\section{Conclusions}

Thyroid cancer survivors have a substantial CHD risk even at long-term follow-up, especially in those patients $<65$ years. Further research on the association between thyroid cancer and CHD risk is warranted.

\section{Introduction}


Thyroid cancer was the fifth most common cancer among women and the ninth most common cancer among both sexes in 2018. The incidence has dramatically increased worldwide over recent decades ${ }^{1}$ with an $211 \%$ increase in the United States between 1975 and $2013^{2}$ (2) and 490\% in South Korea between 1995 and $2005^{3}$, although thyroid cancer-related mortality rates have not changed substantially ${ }^{4}$. Initial thyroid cancer treatment comprises total thyroidectomy, radioactive iodine therapy, and administration of exogenous thyroxin to facilitate thyroid stimulating hormone suppressive. ${ }^{4}$ Angus, et al. 5 .

Radioactive iodine and thyroid stimulating hormone suppressive therapies can lead to short-term adverse events, such as thyroiditis, hypothyroidism and hyperthyroidism related palpitation and progressive atherosclerosis in the carotid intima ${ }^{6}$. Although several studies had consistently the short-term cardiovascular effect, the long-term risk of coronary heart disease (CHD), ischemic stroke (IS), and atrial fibrillation $(\mathrm{AF})$ in individuals with thyroid cancer remains controversial.

For example, some studies have demonstrated that thyroid cancer patients had higher odds (ranging from 1.05 to 1.28) of developing cardiovascular disease ${ }^{7,8}$. However, another research failed to find an association between thyroid cancer and the risk of cardiovascular disease ${ }^{9}$. Furthermore, some researcher found an increased risk (ranging from 1.06 to 3.95) of AF among thyroid cancer patients ${ }^{7,10}$, while another study did not find any such association ${ }^{11}$. Using a nationwide population-based cohort, we aimed to examine the following hypotheses: (1) CHD and IS are more frequent in thyroid cancer patients than in the general reference population, (2) AF is as frequent in thyroid cancer patients as in the general reference population, and (3) the risk of cardiovascular disease among thyroid cancer patients was the same across different ages.

\section{Materials And Methods}

This retrospective cohort study used data from the National Taiwan Cancer Registry Database (NTCRD). The data analysis was conducted for the period from January 1, 2011 to December 31, 2016 and followed up until December 31, 2018. The NTCRD was set up in 1979 to collect data of all confirmed cancer diagnoses among Taiwanese citizens since 2002, and it has been revised for the third time to date. The NTCRD contains cancer characteristics including staging; first-time surgical treatment; chemo-, radiation, hormone, and target therapy; and lifestyle habits such as smoking and alcohol and betel nut consumption. Additionally, the NTCRD was merged with the National Health Insurance Research Database (NHIRD) and national death records. The NHIRD incorporated longitudinal information of $99 \%$ citizens' demographic, medical, and pharmaceutical data based on the International Classification of Disease, 9th revision (ICD-9), medical procedure data, hospitalization and outpatient clinic records, and drug prescriptions. The national death records list the specific causes of death based on the ICD-9/ICD-10 codes. Additional details on the cohort protocol are available in a previously published study ${ }^{12}$. The protocol was reviewed and approved by the Research Ethics Committee of Mackay Memorial Hospital. 
For the present analysis, baseline was defined as the day when the thyroid cancer patients received thyroidectomy in the period from January 1, 2011 to December 31, 2016. From all the patients who underwent thyroidectomy, we excluded those participants who were $<20$ or $>85$ years old, had cancers other than thyroid cancer, and had history of CHD, IS, or AF before index date (362 with cancers other than thyroid cancer, 101 with CHD, 32 with IS, and 128 with AF) (Supplementary Figure 1).

\section{Assessment of coronary heart disease, ischemic stroke, atrial fibrillation, and cardiovascular diseases}

After thyroid cancer diagnosis, patients were followed up until December 31, 2018. The endpoint of primary interest among thyroid cancer patients and the general reference population was hospitalization for CHD, defined as fatal and non-fatal CHD (including non-fatal myocardial infarction and coronary artery bypass graft surgery) after thyroid cancer diagnosis in NHIRD claim records based on ICD-9 (Table 1). Fatal CHD was defined as death caused by $\mathrm{CHD}$ as listed in the death certificate on the national death records. Secondary interest included IS defined by one hospitalization diagnosis and AF defined by one hospitalization or two outpatient clinic diagnoses after thyroid cancer was diagnosed by ICD-9. The definition of cardiovascular diseases was CHD or IS. 
Table 1

Baseline characteristics of Taiwan thyroid cancer cohort patients without coronary heart disease at baseline

\begin{tabular}{|lll|}
\hline & N & $\%$ \\
\hline All & 4274 & 100 \\
\hline Mean age (SD) & 49 & 13.1 \\
\hline Sex & & \\
\hline Men & 1041 & 24.4 \\
\hline Women & 3233 & 75.6 \\
\hline BMI (kg/m ${ }^{2}$ ) & & \\
\hline$<18.5$ & 156 & 3.8 \\
\hline 18.5-23.9 & 1874 & 46 \\
\hline $24.0-26.9$ & 1074 & 26.4 \\
\hline$\geqq 27.0$ & 971 & 23.8 \\
\hline Urbanization & & \\
\hline Urban & 3267 & 76.4 \\
\hline Non-urban & 1007 & 23.6 \\
\hline Current smoking or quit < 15 years & 366 & 8.6 \\
\hline TNM staging & & \\
\hline Stage I & 2865 & 68.6 \\
\hline Stage II & 248 & 5.9 \\
\hline Stage III & 754 & 18 \\
\hline Stage IV & 312 & 7.5 \\
\hline Hypertension & 1814 & 42.4 \\
\hline Diabetes & 473 & 11.1 \\
\hline Hyperlipidemia & & 19.2 \\
\hline Aspirin used & & 9.9 \\
\hline
\end{tabular}


We compared the CHD incidence of Taiwanese thyroid cancer patients with that of the general population using the standardized incidence ratio (SIR), computed as the ratio of observed to expected CHD cases, stratified by age and sex. The incidences of CHD, IS, and AF were compared with the age- and sex-specific disease incidence rates in the Taiwanese population, accounting for person-years of observation. CHD, IS, and $\mathrm{AF}$ incidence data from the NHIRD were used as a reference.

We computed SIRs separately among thyroid cancer patients for both sexes, each age group (20-39, 40$59, \geq 60$ years), and different follow-up year and compared them with that of the reference population. The change in trends of SIRs for both sexes and age $<65$ or $\geq 65$ years old in different follow-up years was analyzed. SAS version 9.4 (SAS Institute, Cary, NC, USA) and Stata version 12 (Stata Corporation, College Station, TX, USA) were used for statistical analyses.

\section{Results}

\section{Sample characteristics}

After excluding 128 individuals who were $<20$ or $>85$ years old or had missing index data, 4375 thyroid cancer patients were eligible for the study. Further, 4274 patients without CHD history, 4343 patients without IS history, and 4247 patients without AF history were included for CHD, IS, and AF analysis, respectively. The mean age was 49 (standard deviation, 13.1) years and mean follow-up was 3.5 (standard deviation, 1.2) years. The basic characteristics of the thyroid cancer patients in the study are shown in Table 2.

Table 2

Cardiovascular incidence, displayed as standardized incidence ratios (SIR) and 95\% confidence intervals subdivided by three cardiovascular endpoints and atrial fibrillation

\begin{tabular}{|lllll|}
\hline Primary Endpoints & Events & SIR & $95 \%$ Cl & \\
\hline CHD & & & Lower & Upper \\
\hline IS & 70 & 1.57 & 1.2 & 1.93 \\
\hline AF & 30 & 0.74 & 0.47 & 1 \\
\hline CVD & 20 & 0.74 & 0.42 & 1.06 \\
\hline $\begin{array}{l}\text { Abbreviations: CHD, coronary heart disease; IS, ischemic stroke; CVD, cardiovascular disease; Af, atrial } \\
\text { fibrillation; SIR, standardized incidence ratios; Cl, confidence interval. }\end{array}$ & \\
\hline
\end{tabular}

\section{Observed and expected coronary heart disease and ischemic stroke events}

SIR was significantly high for $\mathrm{CHD}$ (SIR, 1.57; 95\% confidence interval [CI], 1.2 to 1.93 ) and low (not significantly) for IS (SIR, $0.74 ; 95 \% \mathrm{Cl}, 0.47$ to 1$)$, AF (SIR, $0.74 ; 95 \% \mathrm{Cl}, 0.42$ to 1.06 ), and cardiovascular diseases (SIR, 0.88; 95\% Cl, 0.7 to 1.05) (Table 2 and Figure 1). The overall CHD SIRs gradually declined, 
but they were persistent for more than 5 years after the diagnosis of thyroid cancer (Table 3). Although SIRs of CHD were significantly high for both sexes, patients with thyroid cancer at a younger age had higher SIRs than patients with thyroid cancer at $>65$ years of age. Patients $<65$ years of age had a $1.5-$ to 2.66-fold significantly higher CHD SIR, while those $>65$ years of age had 0.57 - to 1.42 -fold odds of CHD SIRs, which remained high, although not significantly (Table 3). The difference in the risk of CHD between the two age groups remained up to 5 years after thyroid cancer diagnosis (Table 4 and Figure 2).

Table 3

Standardized incidence ratio by potential explanatory factors for coronary heart disease

\begin{tabular}{|c|c|c|c|c|}
\hline \multicolumn{5}{|c|}{ (A) } \\
\hline \multirow[t]{2}{*}{ Subgroup } & \multirow[t]{2}{*}{ Incidence events } & \multirow[t]{2}{*}{ SIR } & \multicolumn{2}{|l|}{$95 \% \mathrm{Cl}$} \\
\hline & & & Lower & Upper \\
\hline All & 70 & 1.57 & 1.2 & 1.93 \\
\hline \multicolumn{5}{|l|}{ Age at diagnosis } \\
\hline$<65$ & 49 & 2.08 & 1.5 & 2.66 \\
\hline$\geqq 65$ & 21 & 1 & 0.57 & 1.42 \\
\hline \multicolumn{5}{|l|}{ Sex } \\
\hline Men & 28 & 1.63 & 1.03 & 2.24 \\
\hline Men: 20 39 & 2 & 1.25 & 0 & 3.7 \\
\hline Men: 40 64 & 20 & 1.62 & 0.93 & 2.32 \\
\hline Men: $\geqq 65$ & 6 & 0.78 & 0.1 & 1.47 \\
\hline Women & 42 & 1.53 & 1.06 & 1.99 \\
\hline Women: 20 39 & 1 & 1.8 & 0 & 5.33 \\
\hline Women: 40 64 & 26 & 1.59 & 0.98 & 2.2 \\
\hline Women: $\geqq 65$ & 15 & 1.01 & 0.5 & 1.53 \\
\hline \multicolumn{5}{|c|}{ Follow-up (years) } \\
\hline 1 & 27 & 2.05 & 1.28 & 2.83 \\
\hline 2 & 42 & 1.65 & 1.15 & 2.15 \\
\hline 3 & 53 & 1.5 & 1.09 & 1.9 \\
\hline 4 & 67 & 1.6 & 1.22 & 1.99 \\
\hline 5 & 70 & 1.57 & 1.2 & 1.93 \\
\hline
\end{tabular}


Table 4

Standardized incidence for coronary heart disease at different follow up years stratified by age younger than 65 years old and older than 65

\begin{tabular}{|c|c|c|c|c|c|c|c|c|}
\hline \multirow[b]{2}{*}{$\begin{array}{l}\text { Follow-up } \\
\text { (years) }\end{array}$} & \multicolumn{4}{|c|}{ Age $<65$ years old } & \multicolumn{4}{|c|}{ Age $>=65$ years old } \\
\hline & $\begin{array}{l}\text { Incidence } \\
\text { events }\end{array}$ & SIR & $95 \% \mathrm{Cl}$ & & $\begin{array}{l}\text { Incidence } \\
\text { events }\end{array}$ & SIR & $95 \% \mathrm{Cl}$ & \\
\hline & & & Lower & Upper & & & Lower & Upper \\
\hline 1 & 16 & 2.54 & 1.3 & 3.79 & 11 & 1.6 & 0.66 & 2.55 \\
\hline 2 & 28 & 2.23 & 1.4 & 3.05 & 14 & 1.09 & 0.52 & 1.66 \\
\hline 3 & 36 & 1.99 & 1.34 & 2.64 & 17 & 0.98 & 0.51 & 1.45 \\
\hline 4 & 47 & 2.17 & 1.55 & 2.79 & 20 & 1 & 0.56 & 1.43 \\
\hline 5 & 49 & 2.08 & 1.5 & 2.66 & 21 & 1 & 0.57 & 1.42 \\
\hline
\end{tabular}

Observed And Expected Ischemic Stroke And Atrial Fibrillation Events

Patients with thyroid cancer had lower risk (not significant) of IS and AF than the general reference population. The SIR for IS was lower (not significant) than expected (SIR, $0.74 ; 95 \% \mathrm{Cl}, 0.47$ to 1 ) (Table 3 ), while IS risk was significantly lower among those with thyroid cancer at $>65$ years of age (SIR, 0.6 ; $95 \% \mathrm{Cl}, 0.27$ to 0.92 ) (Table 4). SIR was lower (not significant) for AF up to 5 years of follow-up based on the data of 20 participants (SIR, $0.74 ; 95 \% \mathrm{Cl}, 0.42$ to 1.06 ) (Table 2 ).

\section{Discussion}

The analysis of the nationally representative cohort showed that patients with thyroid cancer had higher risk of developing CHD than the general population, although the results were not significant for IS and AF. The CHD risk was highest in young thyroid cancer patients $<65$ years of age, which persisted more than 5 years after cancer diagnosis, while it was the same in patients $>65$ years and the general population.

The results of the present study are in accordance with the findings from a previous study ${ }^{8}$ that showed the risk of $\mathrm{CHD}$ events in patients with thyroid cancer is higher than that in the general population. To the best of our knowledge, no study has conducted SIR analysis for CHD risk among different age groups of thyroid cancer patients. A cohort study of 182419 patients found increased incidence in CHD in thyroid cancer patients without a subgroup analysis of different age stratification ${ }^{8}$. A cohort study of 3706 thyroid cancer patients conducted subgroup analysis of CHD risk in cancer patients of $<40$ and $\geq 40$ years of age and a healthy control. Although higher incidence of CHD was observed among the thyroid cancer patients, the odds were higher in the younger group ( $<40$ years) than those in older population ( $\geq 40$ years) during the 1 to 5 year and 5 to 10 year follow-up periods, and it was not observed in the $>10$ year follow-up ${ }^{11}$. Another cohort study of 6900 patients that conducted age-based subgroup analysis of 
$\mathrm{AF}$ instead of $\mathrm{CHD}$ reported that $\mathrm{AF}$ risk was higher among younger patients than that in older patients. The SIRs of AF was $2.67(95 \% \mathrm{Cl}, 1.61$ to 4.41$)$ in patients $<45$ years old and 1.03 ( $95 \% \mathrm{Cl}, 0.75$ to 1.41$)$ in patients $\geq 75$ years old 9 . These findings suggest that there is a higher long-term risk of $\mathrm{CHD}$ in younger patients than in older patients. In addition, our results demonstrated that the incidence of AF in thyroid cancer patients was similar to that in the general population, which is consistent with a previously published population-based study ${ }^{11}$ and an over-all meta-analysis result. However, in contrast to our findings, a previous study reported a significant 1.66 -fold higher risk of hospitalization for $A^{9,10}$. The discrepancy between the results of the current study and the previous study may be attributed to several reasons. First, in our study, $90 \%$ of the participants were $<60$ years old, while in the previous study onethird of the patients were $>60$ years old. Young patients might be able to tolerate the effect of subclinical thyrotoxicosis, especially after thyroid stimulating hormone suppression therapy. Second, the period of study inclusions was different in the two cohorts. In our study, thyroid cancer patients diagnosed after 2011 were included, while in the previous study, those diagnosed from 1987 to 2013 were included. Decades ago, the prevalent guidelines suggested lifelong thyroid stimulating hormone suppression treatment for all thyroid cancer patients. However, according to the current recommendations, such as the American Thyroid Association guidelines, thyroid stimulating hormone suppression treatment is not indicated for management of low recurrent risk thyroid cancer ${ }^{13}$. Thus, the fewer number of patients with subclinical thyrotoxicosis status could have further reduced the incidence of AF in recent years. This suggests that the recent update of recommendations on long-term AF prevention during thyroid cancer treatment may have resulted in the inconsistent results between the two studies with different periods of inclusion.

Although the pathophysiological reason of increased CHD incidence in thyroid cancer patients remains unclear, several factors might contribute to the higher risk. First, during thyroid cancer treatment, such as thyroidectomy, radioactive iodine therapy, and thyroid stimulating hormone suppression therapy, adverse events of hypothyroidism and hyperthyroidism status may develop. These events are involved in the development of atherosclerosis, a direct link to ischemic heart disease. For example, studies have reported that subclinical hyperthyroidism might contribute to increased left ventricular size ${ }^{14,15}$ and elevated systolic pressure, which reduce arterial elasticity and aggravate diastolic dysfunction ${ }^{16}$. Furthermore, hypercoagulability, a consequence of the pro-thrombotic effects of subclinical hyperthyroidism can also lead to atherosclerosis development. Second, radioactive iodine exposure might induce arterial atherosclerosis. Animal studies have demonstrated that radioactive iodine can cause atherosclerotic-like plaque formation through endothelial proliferation, chronic fibrosis of the intima and media, and occlusive changes in the vasa vasorum ${ }^{17-19}$. Furthermore, the intima-media thickness increased significantly after radioactive iodine ablation in hyperthyroidism patients, indicating the association of progressive atherosclerosis with radioiodine exposure ${ }^{6}$. However, the discrepancy in the odds for CHD and IS incidence in our study, which showed that thyroid cancer patients had a significantly higher risk of fatal and non-fatal CHD but insignificant risk of IS, may be attributed to the different etiologies of the two conditions. In contrast to the homogenous pathophysiology of CHD, where atherosclerosis is the major cause of heart attack, the etiology of IS is more complex. Besides 
atherosclerosis, research showed that cardio-embolism, small-vessel occlusion, non-atherosclerotic vasculopathies, hypercoagulable states, or hematologic disorders could lead to IS events ${ }^{20}$. The heterogeneous etiology results in a more complex association between thyroid cancer and IS.

Our results support the recent recommendations ${ }^{13}$ and have important implications in cardiotoxicity prevention. From a public health perspective, considering cancer as a chronic disease with safe long-term treatment rather than a fatal disease with aggressive intervention may provide pragmatic benefits to cancer patients. Further evidence of the association between radioactive iodine and thyroid stimulating hormone suppression therapies and CHD should be obtained by epidemiology studies.

\section{Limitations}

This study has several limitations. First, the relatively short follow-up period limited our ability to detect cardiovascular events and their association with thyroid cancer. However, the inclusion period of thyroid cancer diagnosis allowed the inclusion of the recent recommendations for thyroid cancer management rather than the recommendations prevalent decades ago. Second, although we conducted SIR analysis using age and sex stratification, residual confounding could have been present in our study, such as smoking and history of hypertension, diabetes, and hyperlipidemia. To deal with this, we conducted subgroup-stratified analyses of SIR and the percentage of basic characteristics in our inclusion population. Third, generalizing our results to other ethnic populations or countries should be treated with caution due to the potential differences in the health care systems and resources. Finally, the study findings are observational and cannot be used to establish causality, and further research is warranted.

\section{Conclusion}

In conclusion, while there were no significant risks for IS and AF, thyroid cancer survivors, especially young patients, have a significant risk of developing CHD that persists even at long-term follow-up. Further research on the association between thyroid cancer and the odds of $\mathrm{CHD}$, including factors such as diagnosed age, cancer characteristics, and management, should be conducted.

\section{Declarations}

\section{Acknowledgments}

The datasets generated and/or analysed during the current study are not publicly available due to the terms of consent to which the participants agreed but data are however available from the authors upon reasonable request and with permission of the Taiwanese National Health Insurance Research Database of Ministry of Health and Welfare (NHIRD_MOHW), Taiwan Cancer Registry Center and Ministry of Science and Technology (MOST 110-2314-B-195-004)..

\section{Financial Support and Author disclosures:}


This paper was supported by grants from the Health Promotion Administration, Ministry of Health and Welfare (A1091115 Tobacco Health and Welfare Taxation) in

Taiwan. The content of this research may not represent the opinion of the Health Promotion Administration, Ministry of Health and Welfare in Taiwan. The funders had no role in the study design, data collection and analysis, decision to publish, or preparation of the manuscript. M.-C., T. is part-funded by Ministry of Science and Technology (MOST 110-2314-B-195-004). All the other authors have no relationships with any company that might have an interest in the submitted work in the previous 3 years. Their spouses, partners, or children have no financial relationships that may be relevant to the submitted work; and all the authors have no non-financial interests that may be relevant to the submitted work.

\section{Author Contribution}

M.-C.T., W.-C.L., C.-J.C., P.-J.T. and K.-L.C. designed the study. M.-C.T., W.-H.T., P.-J.T. and K.-L.C. contributed to writing of the manuscript. M.-C.T., C.-T. H., H.-Y. H. and T.-L. Y. contributed software and formal analysis. W.-C.L., C.-J.C. and K.-L.C. had project administration and data resources. W.-C.L., B.-Y.H., J.R.J, and C.-J.C. contributed to collection and/or assembly of data. M.-C.T., W.-H.T., S.-P.C., C.-L.C., C.C.L., S.-C.L. and P.-J.T. contributed to interpretation. M.-C.T. and K.-L.C. had revisions and final approval of the manuscript. K.-L.C. is the guarantor of this work and, as such, had full access to all the data in the study and takes responsibility for the integrity of the data and the accuracy of the data analysis.

\section{Author Disclosure Statement}

All other authors have no potential conflicts of interest relevant to this article.

\section{Funding statement}

The funding agencies had no role in study design, data collection and analysis, decision to publish, or preparation of the manuscript.

\section{Ethics approval and consent to participate:}

The Mackay Memorial Hospital Committee Review Board approved the study protocol (IRB $20 \mathrm{MMHIS} 475 \mathrm{e}$ approved with exempt review). The authors confirm that all of the research meets the ethics guidelines, including adherence to the legal requirements of the country where the study was performed.

\section{References}

1. Vaccarella, S. et al. The Impact of Diagnostic Changes on the Rise in Thyroid Cancer Incidence: A Population-Based Study in Selected High-Resource Countries. Thyroid: official journal of the American Thyroid Association, 25, 1127-1136 https://doi.org/doi:10.1089/thy.2015.0116 (2015).

2. Institute, N. C. Previous Version: SEER Cancer Statistics Review, 1975-2013. (2016) 
3. Vaccarella, S. et al. Worldwide Thyroid-Cancer Epidemic? The Increasing Impact of Overdiagnosis. The New England journal of medicine, 375, 614-617 https://doi.org/doi:10.1056/NEJMp1604412 (2016).

4. World Cancer Research Fund, A. I. f. C. R. Worldwide cancer data:Global cancer statistics for the most common cancers

5. Angus, D. C. et al. Effect of Hydrocortisone on Mortality and Organ Support in Patients With Severe COVID-19: The REMAP-CAP COVID-19 Corticosteroid Domain Randomized Clinical Trial., 324, 13171329 https://doi.org/doi:10.1001/jama.2020.17022 (2020).

6. Sürücü, E. et al. The effect of radioiodine on the intima media thickness of the carotid artery. Molecular imaging and radionuclide therapy, 22, 85-89 https://doi.org/doi:10.4274/Mirt.24119 (2013).

7. Pajamäki, N. et al. Long-term cardiovascular morbidity and mortality in patients treated for differentiated thyroid cancer. Clinical endocrinology, 88, 303-310 https://doi.org/doi:10.1111/cen.13519 (2018).

8. Suh, B. et al. Increased cardiovascular risk in thyroid cancer patients taking levothyroxine: a nationwide cohort study in Korea. European journal of endocrinology, 180, 11-20 https://doi.org/doi:10.1530/eje-18-0551 (2019).

9. Zoltek, M., Andersson, T. M., Hedman, C., Ihre-Lundgren, C. \& Nordenvall, C. Cardiovascular Incidence in 6900 Patients with Differentiated Thyroid Cancer: a Swedish Nationwide Study. World journal of surgery, 44, 436-441 https://doi.org/doi:10.1007/s00268-019-05249-8 (2020).

10. Klein Hesselink, E. N. et al. Increased Risk of Atrial Fibrillation After Treatment for Differentiated Thyroid Carcinoma. The Journal of clinical endocrinology and metabolism, 100, 4563-4569 https://doi.org/doi:10.1210/jc.2015-2782 (2015).

11. Blackburn, B. E. et al. Aging-Related Disease Risks among Young Thyroid Cancer Survivors. Cancer epidemiology, biomarkers \& prevention: a publication of the American Association for Cancer Research. cosponsored by the American Society of Preventive Oncology, 26, 1695-1704 https://doi.org/doi:10.1158/1055-9965.Epi-17-0623 (2017).

12. Kao, W. H. et al. Validity of cancer diagnosis in the National Health Insurance database compared with the linked National Cancer Registry in Taiwan. Pharmacoepidemiology and drug safety, 27, 1060-1066 https://doi.org/doi:10.1002/pds.4267 (2018).

13. Haugen, B. R. et al. 2015 American Thyroid Association Management Guidelines for Adult Patients with Thyroid Nodules and Differentiated Thyroid Cancer: The American Thyroid Association Guidelines Task Force on Thyroid Nodules and Differentiated Thyroid Cancer. Thyroid: official journal of the American Thyroid Association 26, 1-133, doi:10.1089/thy.2015.0020 (2016)

14. Biondi, B., Palmieri, E. A., Lombardi, G. \& Fazio, S. Effects of thyroid hormone on cardiac function: the relative importance of heart rate, loading conditions, and myocardial contractility in the regulation of cardiac performance in human hyperthyroidism. The Journal of clinical endocrinology and metabolism, 87, 968-974 https://doi.org/doi:10.1210/jcem.87.3.8302 (2002). 
15. Biondi, B. et al. Endogenous subclinical hyperthyroidism affects quality of life and cardiac morphology and function in young and middle-aged patients. The Journal of clinical endocrinology and metabolism, 85, 4701-4705 https://doi.org/doi:10.1210/jcem.85.12.7085 (2000).

16. Boswijk, E. et al. TSH suppression aggravates arterial inflammation - an (18)F-FDG PET study in thyroid carcinoma patients. European journal of nuclear medicine and molecular imaging, 46, 14281438 https://doi.org/doi:10.1007/s00259-019-04292-w (2019).

17. Murros, K. E. \& Toole, J. F. The effect of radiation on carotid arteries. A review article. Archives of neurology, 46, 449-455 https://doi.org/doi:10.1001/archneur.1989.00520400109029 (1989).

18. Hopewell, J. W. et al. Vascular irradiation damage: its cellular basis and likely consequences. The British journal of cancer. Supplement, 7, 181-191 (1986).

19. Cheng, S. W., Ting, A. C. \& Wu, L. L. Ultrasonic analysis of plaque characteristics and intimal-medial thickness in radiation-induced atherosclerotic carotid arteries. European journal of vascular and endovascular surgery: the official journal of the European Society for Vascular Surgery, 24, 499-504 https://doi.org/doi:10.1053/ejvs.2002.1752 (2002).

20. Adams, H. P. Jr. et al. Classification of subtype of acute ischemic stroke. Definitions for use in a multicenter clinical trial. TOAST. Trial of Org 10172 in Acute Stroke Treatment., 24, 35-41 https://doi.org/doi:10.1161/01.str.24.1.35 (1993).

\section{Figures}

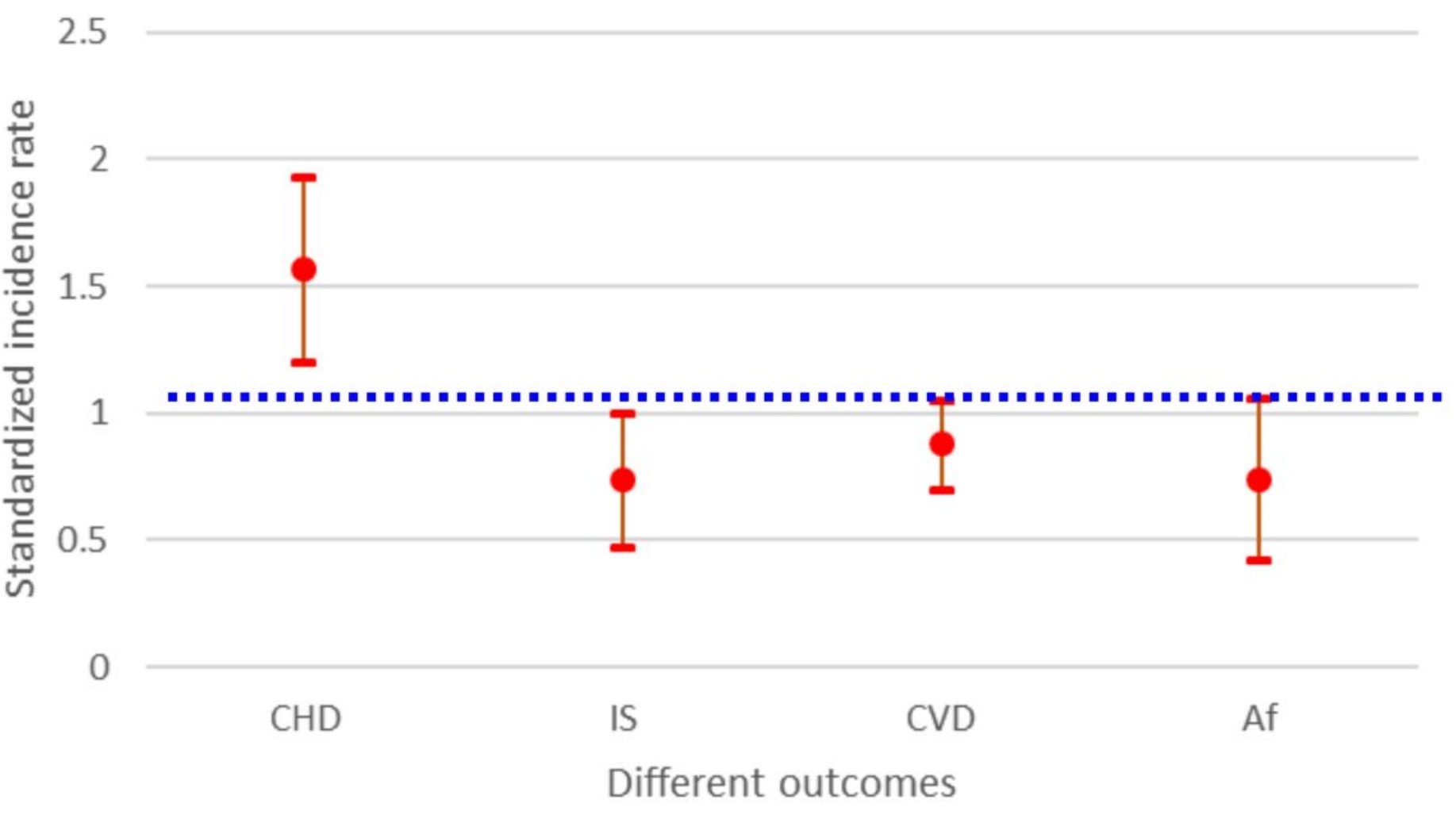


Figure 1

Cardiovascular and atrial fibrillation incidence, displayed as standardized incidence ratios (SIR) and 95\% confidence intervals subdivided by five cardiovascular and atrial fibrillation endpoints. The blue line crossing SIR 1.0 equals the incidence rate of the general population

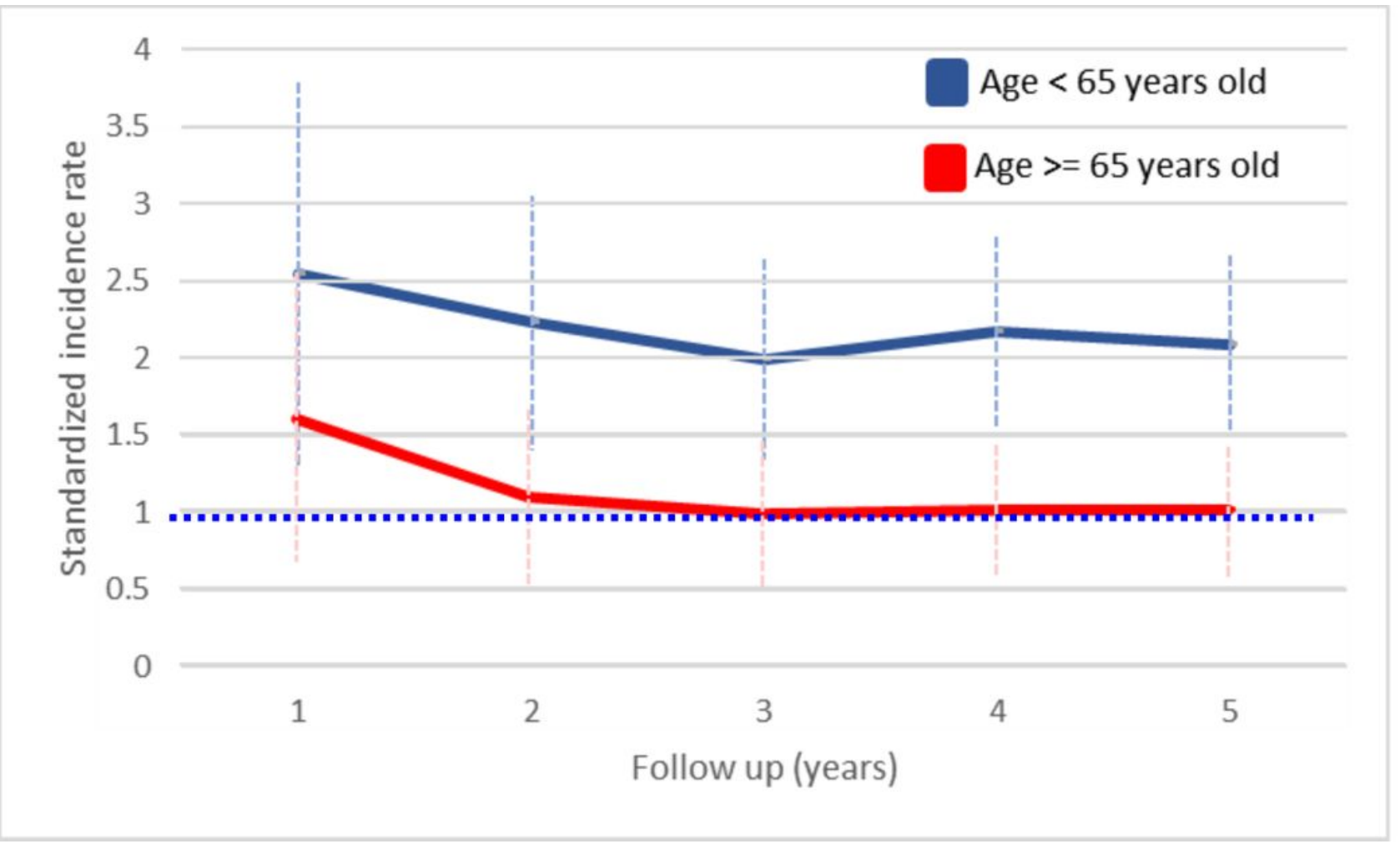

Figure 2

Standardized incidence for coronary heart disease at different follow up years stratified by age younger than 65 years old and older than 65

\section{Supplementary Files}

This is a list of supplementary files associated with this preprint. Click to download.

- Supplement.1113.docx 